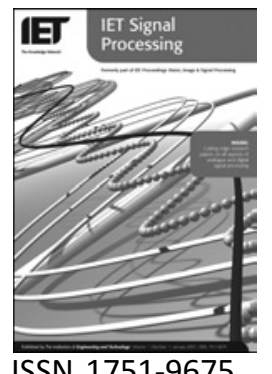

\title{
Signal recovery from partial fractional Fourier domain information and its applications
}

\author{
H.E. Guven ${ }^{1}$ H.M. Ozaktas ${ }^{2}$ A.E. Cetin ${ }^{2}$ B. Barshan ${ }^{2}$ \\ ${ }^{1}$ CenSSIS Headquarters, Northeastern University, 360 Huntington Avenue, Stearns Center Suite 302, Boston, MA 02115, USA \\ ${ }^{2}$ Department of Electrical and Electronics Engineering, Bilkent University, Bilkent, Ankara 06800, Turkey \\ E-mail: eguven@ece.neu.edu
}

\begin{abstract}
The problem of recovering signals from partial fractional Fourier transform information arises in wave propagation problems where the measured information is partial, spread over several observation planes, or not of sufficient spatial resolution or accuracy. This problem can be solved with the method of projections onto convex sets, with the convergence of the iterative algorithm being assured. Several prototypical application scenarios and simulation examples are presented.
\end{abstract}

\section{Introduction}

The fractional Fourier transform (FRT) has found many applications in signal and image processing and optics $[1-15]$. The ath-order FRT operation corresponds to the ath power of the ordinary Fourier transform operation. The zeroth-order FRT of a function is the function itself and the first-order transform is equal to the ordinary Fourier transform. The relationship of the FRT to wave and beam propagation is well established [16-18]. It is well known that the Fourier transform of the original object, aperture or source distribution is observed in the far field. It has been shown that at closer distances, one observes the FRTs of the original object. As the wave propagates, its distribution evolves through FRTs of increasing order. In other words, it is continually fractional Fourier transformed as it propagates, starting from the original function and finally reaching its ordinary Fourier transform in the far field. Thus the problem of recovering signals from partial FRT information naturally finds applications in wave propagation problems where the measured information is partial, spread over several observation planes, or not of sufficient spatial resolution or accuracy.

In this paper, the problem of signal recovery from partial FRT information is solved by using an iterative algorithm involving alternating projections onto sets representing measurements in fractional Fourier domains.
(This problem was first proposed and preliminary results presented in [19].) The reconstruction algorithm is globally convergent and it is based on the method of projections onto convex sets (POCS), a classical numerical technique [20-22]. The convergence of this algorithm can be proved easily for both continuous and discrete signals because partial fractional Fourier information in an interval corresponds to a closed and convex set [in $L^{2}(\mathbb{R})$ and $\ell^{2}$, respectively]. Other closed and convex sets that may be used in the reconstruction algorithm include sets representing bounded energy, the non-negativity constraint and finite-support information in the time or space domain.

The purpose of this paper is to show that partial FRT information distributed over several fractional Fourier domains can be used to reconstruct a signal and to illustrate this with a diversity of application scenarios involving different distributions of the available information. It is not the aim of this paper to determine the best algorithm for this purpose. Our main focus is to provide a framework for consolidating distributed information regarding a wavefield. The POCS algorithm has been used in our simulations for its ease of implementation, but we should note that straightforward application of the POCS procedure may not produce the best convergence rates [22]. Methods for obtaining faster algorithms are discussed and reviewed in [22, 23]. 
In the next section, brief information on the FRT is given. In Section 3, the signal recovery algorithm is described. Section 4 discusses several prototypical application scenarios and simulation examples. In an example from radar signal processing, radar pulses under chirp-type interference are successfully retrieved using the proposed algorithm. In another example inspired by swarm robots/sensor networks, it is shown that a wavefield can be recovered from an irregular array of known samples.

\section{FRT and the signal recovery problem}

In this section, the FRT is briefly reviewed and the signal recovery problem is formulated. For a comprehensive treatment of the transform and its properties the reader is referred to [1]. Let us denote the ath-order FRT operator by $\mathcal{F}^{a}$. When $a=1$ we have the ordinary Fourier transform operator $\mathcal{F}$. The FRT may be defined by standard eigenvalue methods for finding a function $G(\mathcal{H})$ of a linear operator $\mathcal{H}$. We consider the Hilbert space $L^{2}(\mathbb{R})$. Hermite-Gaussian functions are the eigenfunctions of the ordinary Fourier transform

$$
\mathcal{F} \psi_{n}(u)=\exp (-i n \pi / 2) \psi_{n}(u)
$$

where

$$
\begin{aligned}
\psi_{n}(u)= & 2^{1 / 4}\left(2^{n} n !\right)^{-1 / 2} H_{n}(\sqrt{2 \pi} u) \\
& \times \exp \left(-\pi u^{2}\right), \quad n=0,1,2, \ldots
\end{aligned}
$$

are the set of Hermite-Gaussian functions and $H_{n}(u)$ are the standard Hermite polynomials. The FRT is defined in terms of the eigenvalue equation $\mathcal{F}^{a} \psi_{n}(u)=[\exp (-i n \pi / 2)]^{a} \psi_{n}(u)$ with the fractional ath power $[\exp (-i n \pi / 2)]^{a}=\exp (-i a n \pi / 2)$. An analytic expression for the FRT of an arbitrary squareintegrable function $x(u)$ can be obtained by expanding it in terms of the complete orthonormal set of functions $\psi_{n}(u)$ and then applying the above eigenvalue equation to each term of the expansion. This leads to the following expression for the athorder FRT $x_{a}(u) \equiv \mathcal{F}^{a}{ }_{x(u)}[1]$

$$
\begin{aligned}
x_{a}(u)= & {\left[1-i \cot \left(\frac{a \pi}{2}\right)\right]^{1 / 2} \int_{-\infty}^{\infty} \mathrm{d} u^{\prime} x\left(u^{\prime}\right) } \\
& \times \exp \left\{i \pi \left[\cot \left(\frac{a \pi}{2}\right) u^{2}-2 \csc \left(\frac{a \pi}{2}\right) u u^{\prime}\right.\right. \\
& \left.\left.+\cot \left(\frac{a \pi}{2}\right) u^{\prime 2}\right]\right\}
\end{aligned}
$$

The zeroth-order FRT of a function is the function itself and the first-order transform is equal to the ordinary Fourier transform. Positive and negative integer values of a simply correspond to repeated application of the ordinary forward and inverse Fourier transforms, respectively. The fractional Fourier transform operator satisfies index additivity: $\mathcal{F}^{a_{2}} \mathcal{F}^{a_{1}}=\mathcal{F}^{a_{2}+a_{1}}$. The operator $\mathcal{F}^{a}$ is periodic in a with period 4 since $\mathcal{F}^{2}$ equals the parity operator which maps $x(u)$ to $x(-u)$ and $\mathcal{F}^{4}$ equals the identity operator.

Just as it is customary to refer to the domain where the time or space representation of a signal lives as the time or space domain, and the domain where the Fourier transform representation of the signal lives as the frequency domain, we will refer to the domain where the ath FRT $x_{a}(u)$ lives as the ath-order fractional Fourier domain. These domains have a meaningful interpretation in the time- or space-frequency plane. With the time/space domain representing the horizontal axis and the frequency domain representing the vertical axis, the ath domain is the axis making angle $a \pi / 2$ with the horizontal axis [13].

The $a$ th-order discrete FRT $\boldsymbol{x}_{a}$ of an $N \times 1$ vector $\boldsymbol{x}$ is defined as $\boldsymbol{x}_{a}=\boldsymbol{F}^{a} \boldsymbol{x}$, where $\boldsymbol{F}^{a}$ is the $N \times N$ discrete FRT matrix [24, 25], which is essentially the ath power of the ordinary discrete Fourier transform matrix $\boldsymbol{F}$. Let the discrete-time vector $\boldsymbol{x}$ contain the samples $x[n]$ of the continuous-time signal $x(u)$. If $N$ is chosen equal to or greater than the space-bandwidth product of the signal $x(u)$, then the discrete fractional transform approximates the continuous fractional transform in the same way as the ordinary discrete transform approximates the ordinary continuous transform.

The signal recovery problem under consideration is the reconstruction of $x[n]$ from $\hat{x}_{a}[n], n \in U \subset \mathbb{Z}$, at one or more domains $a=a_{1}, a_{2}, \ldots$ Here $\hat{x}_{a}[n]$ denotes the known values of $x_{a}[n]$. The set $U$ may consist of the union of an arbitrary collection of intervals in the ath fractional Fourier domain. It may also contain or consist of isolated points representing measurements $\hat{x}_{a}[n]$ of $x_{a}[n]$ at $n=n_{i}, i=1,2, \ldots$.

\section{Iterative signal recovery algorithm}

This section describes the signal recovery algorithm which is based on the method of POCS that has been successfully used in many signal recovery and restoration problems [23, 26-30]. The theoretical basis of this method may be found in [20-22]. The key idea is to obtain a solution which is consistent with all the available information. In this method, the set of all possible signals is assumed to constitute a Hilbert space with an associated norm in which the 
prior information about the desired signal can be represented in terms of convex sets. In this paper, the Hilbert space is $\ell^{2}$ for discrete-time signals. Let us suppose that the information about the desired signal is represented by $M$ sets, $C_{m}, m=1,2, \ldots, M$. Since the desired signal satisfies all of the constraints it must be in the intersection set $C_{0}=\bigcap_{m=1}^{M} C_{m}$. Any member of the set $C_{0}$ is called a feasible solution [27]. If all of the sets $C_{m}$ are closed and convex then a feasible solution can be found by making successive orthogonal projections onto the sets $C_{m}$. Let $P_{m}$ be the orthogonal projection operator onto the set $C_{m}$. The iterates defined by the following equation

$$
y^{(l+1)}[n]=P_{1} P_{2} \cdots P_{M} y^{(I)}[n], \quad 1=0,1,2, \ldots
$$

converge to a member of the set $C_{0}$, regardless of the initial signal $y^{(0)}[n]$. The order of projections is immaterial. The rate of convergence can be improved by using non-orthogonal projections as well.

We do not devote further space to the underlying mathematical concepts, which can be found in [22]. POCS is an iterative method whose aim is to find a solution satisfying all of the constraints of the numerical problem. An orthogonal projection is made onto one of the constraint sets in each iteration. This ensures that the current iterate satisfies at least this specific constraint. The iterate is then projected to another constraint set in the next iteration. The iterate will now satisfy the new constraint, but may no longer satisfy the previous one; however, it will typically still be closer to satisfying the previous one compared to when it was not yet projected onto the first constraint at all. The iterate is then cyclically projected onto the other constraint sets with similar effect.

Thus while subsequent projections may partly undo the effect of previous ones, the overall effect of cycling through all constraints is to bring the iterate to more closely satisfying all constraints. When the intersection of the constraint sets is not empty, it is intuitively plausible that this procedure has the potential of finding a solution satisfying all the constraints. Each projection 'pulls' the iterate to the constraint set in question, but otherwise is indifferent to whether the iterate satisfies the other constraints or not. Therefore after multiple rounds of repeated pulling, the iterate ends up in the non-empty solution set. It has been proved [20-22] that the iterative process actually converges to a solution satisfying all the requirements of the problem, if they are represented as closed and convex sets in a Hilbert space.

Let $x_{a_{1}}[n]$ be the discrete FRT of $x[n]$ in the $a_{1}$ th domain. We define the set $C_{1}$ in $\ell^{2}$ as the set of signals whose $a_{1}$ th discrete FRTs are equal to $\hat{x}_{a_{1}}[n]$ for $n \in U_{1}$ in the $a_{1}$ th fractional domain

$$
C_{1}=\left\{x[n]: x_{a_{1}}[n]=\hat{x}_{a_{1}}[n], \quad n \in U_{1}\right\}
$$

This set is convex because the FRT is linear. The proof of closedness can be established as in [20]. If data are also available in another $\left(a_{2}\right.$ th) fractional domain, another set $C_{2}$ can be defined in a similar manner and so on. If the signal is a finite-extent signal, then this information can be modelled as a closed and convex set as in other well-known signal recovery problems. Actually, time/ space-domain information about the original signal such as the knowledge that $x[n]=0$ in a bounded or unbounded window in the time/space domain already belongs to the above class of sets since the time/spacedomain merely corresponds to the special case of $a=0$. In this case, (3) simply becomes the identity operator for the fraction $a=0$.

Another convex set which can be used in the signal recovery algorithm is the bounded energy set $C_{\mathrm{e}}$, which is the set of sequences whose energy is bounded by $\boldsymbol{\epsilon}_{0}$

$$
C_{\mathrm{e}}=\left\{x[n]: \sum_{n}|x[n]|^{2} \leq \epsilon_{0}\right\}
$$

This set provides robustness against noise, if $\boldsymbol{\epsilon}_{0}$ is known or some idea about $\epsilon_{0}$ is available. Yet other convex sets describing partial fractional Fourier domain information can be defined. Non-negativity information about the signal samples also leads to a closed and convex set $C_{p}$ in $\ell^{2}[20,21]$. The same also holds when we know that the signal is real. The set of discrete real signals can be represented by

$$
C_{\mathrm{r}}=\left\{x[n]: x[n]=x^{*}[n], \forall n \in \mathbb{Z}\right\}
$$

where * denotes complex conjugation. The key operation of the method of POCS is the orthogonal projection onto a convex set. Projection operations onto the sets $C_{1}, C_{2}, \ldots$ are straightforward to implement. The orthogonal projection operator onto set $C_{i}$ replaces the FRT values $x_{a_{i}}[n]$ in the index set $U_{i}$, and retains the rest of the data outside the set $U_{i}$

$$
x_{a_{i}}[n] \leftarrow\left\{\begin{array}{ll}
\hat{x}_{a_{i}}[n], & n \in U_{i} \\
x_{a_{i}}[n], & n \notin U_{i}
\end{array} \quad i=1,2, \ldots\right.
$$

where $\hat{x}_{a_{i}}[n]$ represents the known FRT values in the $a_{i}$ th domain.

Orthogonal projection onto the energy set $C_{\mathrm{e}}$ simply consists of scaling the signal $x[n]$ such that the energy of the scaled signal is $\boldsymbol{\epsilon}_{0}$ [22]. Orthogonal projection onto the non-negativity set $C_{\mathrm{p}}$ is carried out by forcing the negative values of $x[n]$ to zero. Orthogonal projection 
onto the realness set $C_{\mathrm{r}}$ is performed by taking the real part of the signal. The formulas for the orthogonal projection operations are summarised below. Projections onto the sets representing partial information in the fractional Fourier domains can be realised as

$$
\begin{gathered}
P_{i} x[n]=F^{-a_{i}} \cdot \begin{cases}\hat{x}_{a_{i}}[n], & n \in U_{i} \\
x_{a_{i}}[n], & n \notin U_{i}\end{cases} \\
i=1,2, \ldots
\end{gathered}
$$

where $\boldsymbol{F}^{-a_{i}}$ is the $-a_{i}$ th discrete FRT operator. Projection onto the bounded-energy set $C_{\mathrm{e}}$ is realised as

$$
P_{\mathrm{e}} x[n]= \begin{cases}x[n], & \boldsymbol{\epsilon} \leq \boldsymbol{\epsilon}_{0} \\ x[n] \cdot \sqrt{\epsilon_{0} / \boldsymbol{\epsilon}}, & \boldsymbol{\epsilon}>\boldsymbol{\epsilon}_{0}\end{cases}
$$

where $\boldsymbol{\epsilon}$ is the energy of $x[n]$. Projection onto the
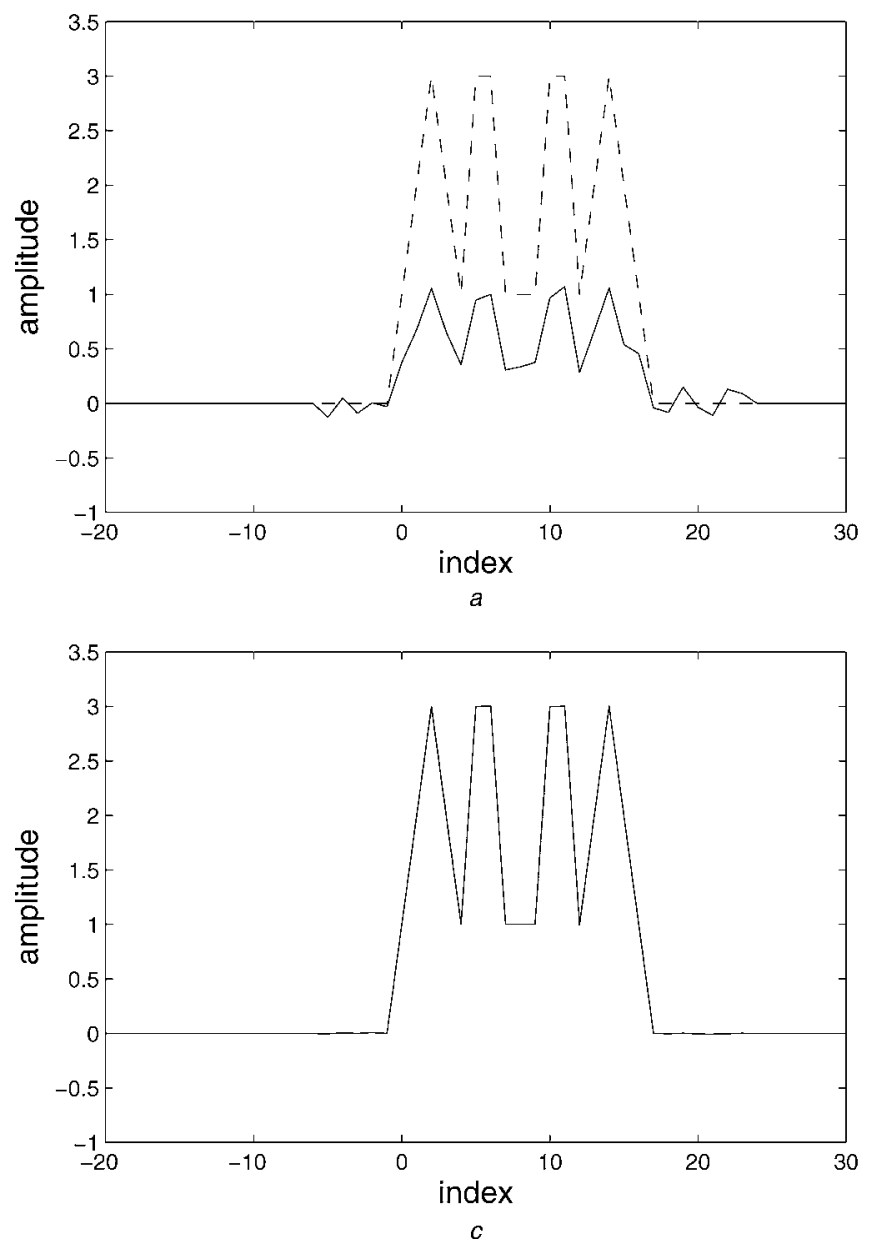

non-negativity set $C_{\mathrm{p}}$ is realised as

$$
P_{\mathrm{p}} x[n]=\left\{\begin{array}{cl}
x[n], & x[n] \geq 0 \\
0, & x[n]<0
\end{array}\right.
$$

Finally, projection onto the real signal set $C_{\mathrm{r}}$ is realised as

$$
P_{\mathrm{r}} x[n]=\frac{x[n]+x^{*}[n]}{2}=\mathfrak{R}(x[n])
$$

Let us now summarise the signal recovery algorithm from partial FRT information.

0 . Set $k=0$ and start with an arbitrary initial estimate $x^{(0)}[n]$.
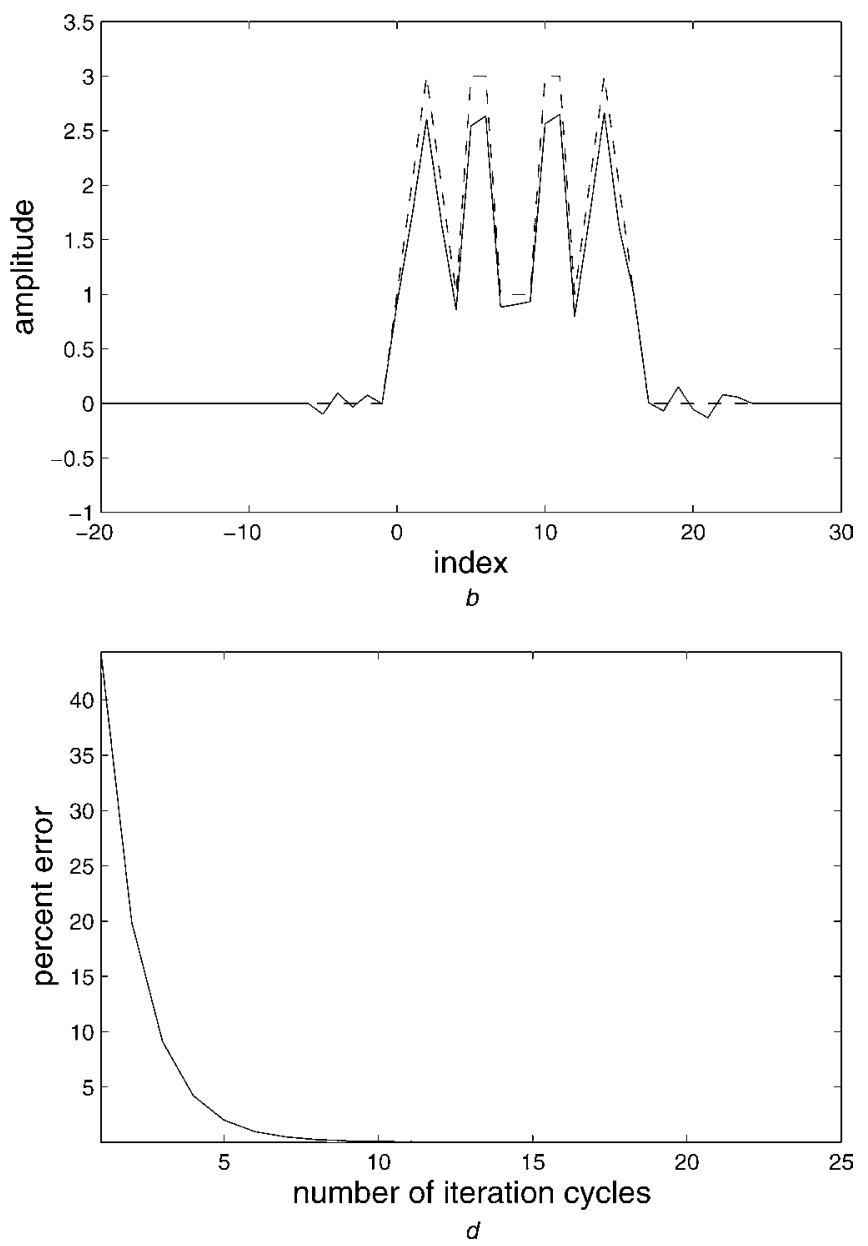

Figure 1 Reconstructed (solid) and original (dashed) signal at the 1st, 5th and 25th iterations and percent error against number of iteration cycles

$a$ Reconstructed (solid) and original (dashed) signal at the 1st iteration

$b$ Reconstructed (solid) and original (dashed) signal at the 5th iteration

$c$ Reconstructed (solid) and original (dashed) signal at the 25th iteration

$d$ Percent error against number of iteration cycles 
1. Project $x^{(k)}[n]$ onto the sets $C_{i}, i=1,2, \ldots$, representing the partial fractional Fourier domain information in domains $a_{i}, i=1,2, \ldots$ [Eq. (9)].

2. If energy, non-negativity, or realness information is available, project onto the corresponding convex sets $C_{\mathrm{e}}, C_{\mathrm{p}}$ or $C_{\mathrm{r}}$ [Eqs. (10)-(12)].

\section{Increase $k$ by 1 .}

4. Stop if $\left\|x^{(k)}[n]-x^{(k-1)}[n]\right\|<\delta$ for some prespecified value of $\delta$, or $k=K$ for a limit $K$ on the number of iterations. Otherwise go to Step 1 .

In our implementation of Step 1, we enumerate the orders $a_{i}$ in increasing order and visit them in this sequence, and we go directly from the $a_{i}$ th order to the $a_{i+1}$ th order with a FRT of order $\left(a_{i+1}-a_{i}\right)$ without having to go back to the zeroth domain. As long as the signal satisfies all of the constraints, the algorithm provides a solution in the set of feasible solutions [20-22].

\section{Application examples}

The problem formulated and solved in this paper is very general and encompasses a variety of application scenarios. In this section, we will present several such prototypical application scenarios and examples; other variations can be easily imagined. To rephrase the general problem, it is assumed that measurements $\hat{x}_{a}[n]$ at $n=n_{i}, i=1,2, \ldots, I_{a}$ for $a=a_{j}, j=1$, $2, \ldots$ are available. This may also include the assumption that the signal is of finite support. Realness information may be additionally available. The FRT integral (3) can either be numerically approximated or, if the fractional Fourier domain data are available on a uniform grid, the problem can be directly posed in terms of the discrete FRT. The latter is assumed in the following examples, all of which are solved according to the POCS procedure described in Section 3.

Scenario 1: Low-resolution version of signal is available in the FRT domain together with finite-extent information [31].

In the examples we consider, it is known that the desired signal is zero outside a certain interval, and only one out of every three samples in the fractional Fourier domain are known over a certain extent.

We assume that the 128-point discrete FRT $x_{0.5}[n]$ of the desired signal $x[n]=\{0, \ldots, 0,1,2$, $3,2,1,3,3,1,1,1,3,3,1,2,3,2,1,0,0, \ldots, 0\}$

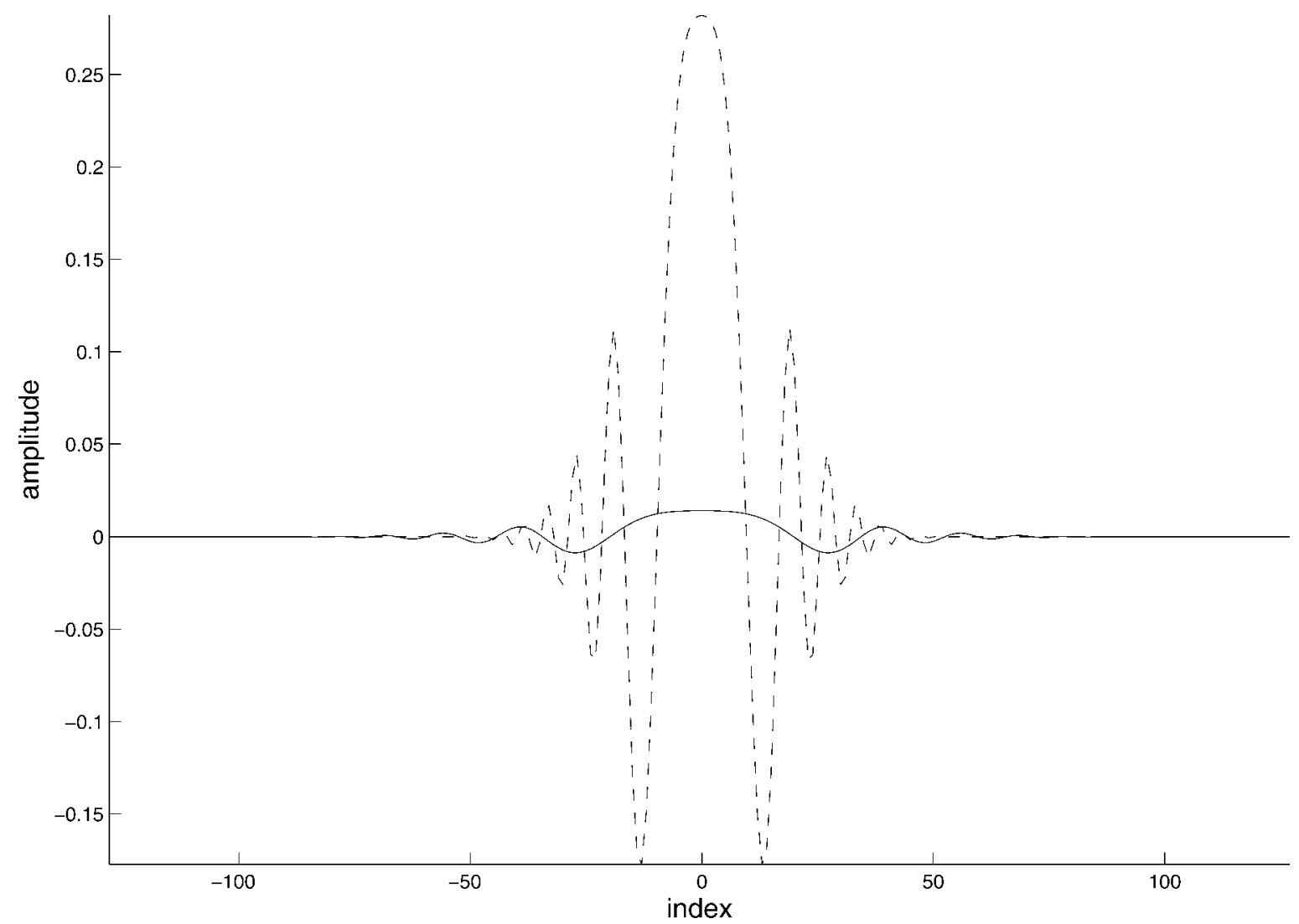

Figure 2 Real parts of the time-domain radar pulse and interference signal

Solid line: radar pulse; dashed line: interference signal 


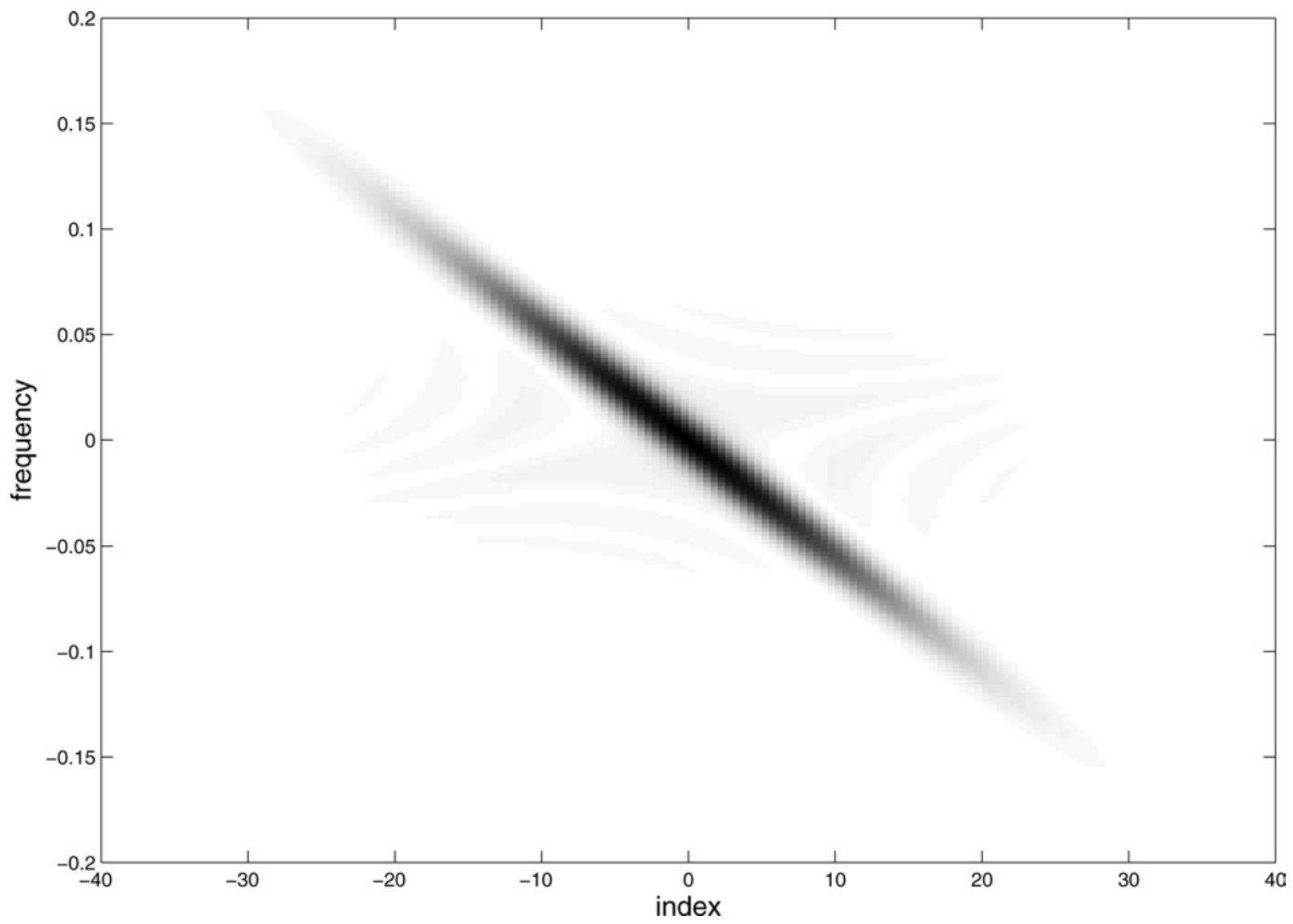

Figure 3 Wigner distribution of the corrupted signal

defined in the interval $-64 \leq n<64$, is available for $n=-64,-61,-58, \ldots, 59,62$. It is also known that $x[n]=0$ outside the interval $-5 \leq n<25$. We define percentage restoration error as follows: $100 \times\left(\sum_{n} \mid x^{(I)}[n]\right.$ $\left.-\left.x[n]\right|^{2}\right) /\left(\sum_{n}|x[n]|^{2}\right)$ where $x^{(I)}[n]$ is the lth iterate. Note that this definition of percentage error involves the energy of the error and not the norm. In all of the examples in this section, the unknown values are initialised to zero. In this example (1a), the percentage error drops below $1 \%$ after 25 iterations.

Let us consider a variation of this example where the available information allows the recovery of the original signal with a much higher accuracy (1b). We assume the fractional Fourier domain information about the original signal is the same as above but the available time-domain information about the signal is that $x[n]=0$ for $n<0$ and $x[n]$ is real everywhere. In this case, the error drops below $0.0001 \%$ after 200 iterations. Figs. $1 a-c$ show the original and reconstructed signals at the end of 1 st, 5 th and 25th iterations. Fig. 1d shows the percent error as a function of the number of iteration cycles.

Let us now consider the same example as in Fig. 1a, but add stationary zero-mean Gaussian white noise onto the measured FRT data, with a signal-to-noise ratio of unity (1c). Because of the substantial amount of noise, the iterates fluctuate around the solution, and the percentage error does not fall below $2 \%$.

Finally, in order to understand the behaviour of the algorithm in the presence of non-uniform samples, we perform the following experiment (1d). The only difference of this example from $1 \mathrm{a}$ is that the samples of $x_{0.5}[n]$ are now assumed to be known at the following randomly selected 40 points in the interval [-64, 64): $n \in\{-59,-57,-53,-49,-48,-45,-44,-43$, $-40,-38,-36,-35,-33,-28,-25,-20,-18$, $-16,-15,-12,2,4,5,8,12,13,20,21,23,25,28$, $33,34,42,56,57,58,59,60,63\}$. The resulting error again falls below 1\% after 25 iterations, suggesting that the performance is not affected significantly by the nonuniform locations of the measurements.

Scenario 2: Low-resolution version of signal is available in two different FRT domains, possibly with additional information [31].

In the examples we consider, only one out of every two samples of the FRT at two or three domains is known. 


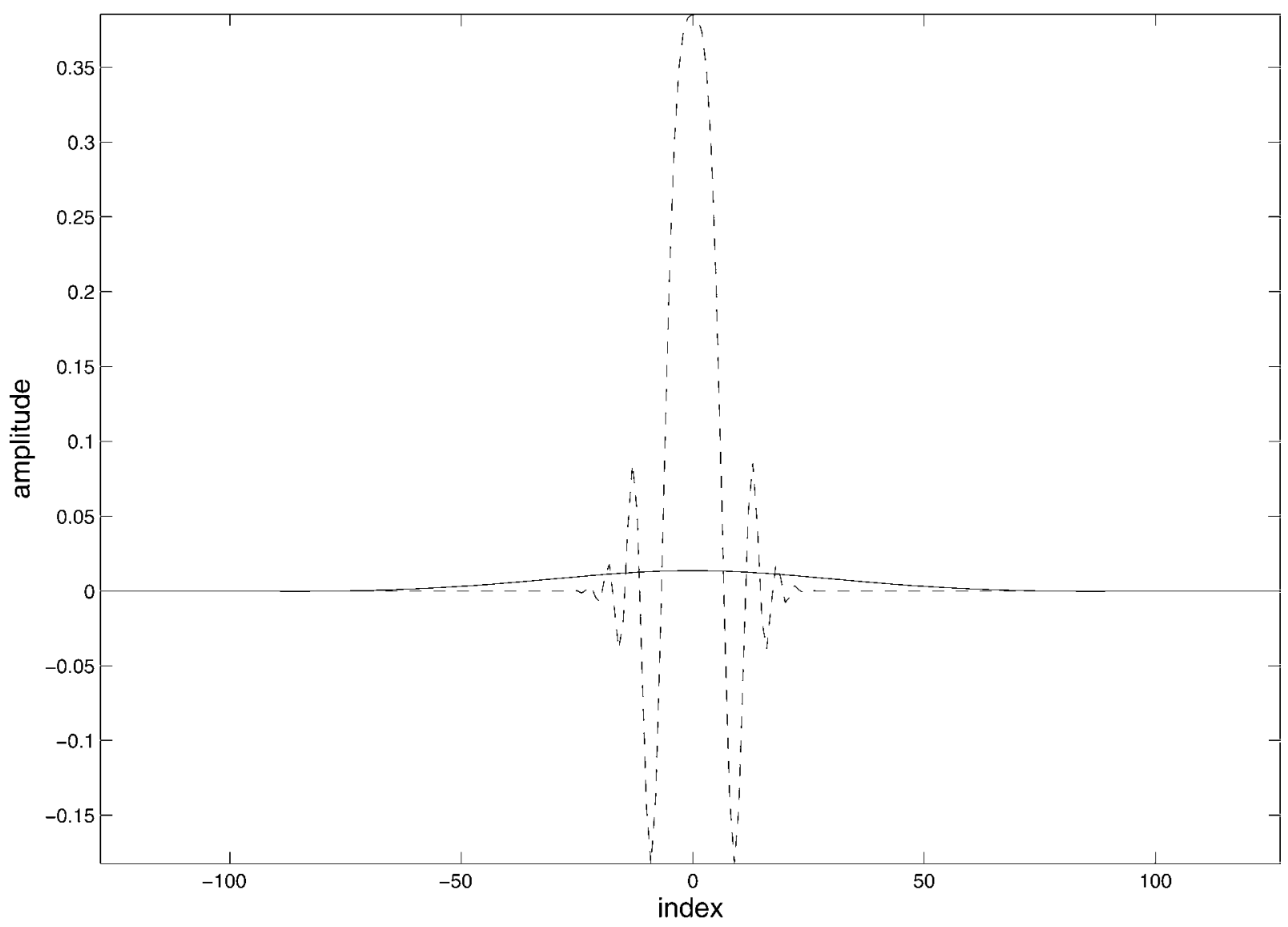

Figure 4 Real parts of the 0.2th fractional Fourier transforms of the radar pulse and interference signal Solid line: radar pulse; dashed line: interference signal

First, we assume that we have all odd samples of $x_{0.5}[n]$ for $-64 \leq n<64$ and all even samples of $x_{0.75}[n]$ for $-64 \leq n<64$. In this example $(2 a)$, the error falls below $1 \%$ after 50 iterations and falls near $0.01 \%$ after 500 iterations.

In the next example (2b), odd samples of $x_{0.5}[n]$ and $x_{0.75}[n]$ are available within a limited range $-25 \leq$ $n<25$. Additionally, we have all even samples of $x[n]$ for $-64 \leq n<64$. This time, after 500 iterations, the error is reduced only to around $2-3 \%$. If we additionally know that the signal is zero outside the interval $-32 \leq n<32$, performance is much improved and in this case (2c), the error falls below $0.0001 \%$ after 100 iterations.

In the final variation of this scenario (2d), we examine the effect of having non-uniformly distributed samples. We assume that both $x_{0.5}[n]$ and $x_{0.75}[n]$ are available for index values of $n \in\{-64,-62, \ldots,-2\} \cup$ $\{0,3, \ldots, 63\}$. That is, we know the value of the signal at one out of every two samples for $n<0$ and at one out of every three samples for $n \geq 0$. We also assume that the signal is zero outside $n \in[-32,32)$. As in $2 \mathrm{c}$, we obtain an error below $0.0001 \%$ after 100 iterations.
Scenario 3: The FRT of the signal is known over a limited interval in a single domain, together with additional constraints.

In the example we consider (3a) the signal is known at full resolution in the $0.3 \mathrm{rd}$ fractional domain over the interval $-15 \leq n<15$. We additionally know that the original signal in the time/space domain is real. The error drops below $0.1 \%$ after 100 iterations.

Scenario 4: The FRT of the signal is known over a limited interval in two or more domains, possibly with additional constraints.

First, we assume (4a) that $x_{0.5}[n]$ is known in the interval $0 \leq n<55$ and $x_{0.75}[n]$ is known in $-55 \leq n<0$. The error drops below $0.1 \%$ after 100 iterations.

Next, we consider the case where the signal is known over a rather small interval in several domains (4b). The signal is known in the $0.2 \mathrm{nd}, 0.3 \mathrm{rd}, 0.5$ th, $0.6 \mathrm{th}, 0.75$ th domains in the interval $-5 \leq n<5$ and we additionally know that $x[n]$ takes real values for all $n$. The error drops below $0.5 \%$ after 100 iterations. 
Finally, to further illustrate the application of the method, we consider the case of a finite-extent radar pulse corrupted by wideband chirp interference. The time-domain radar pulse $x[n]$ and the interference term $y[n]$ are given by

$$
\begin{aligned}
& x[n]=\frac{1}{40 \sqrt{\pi}} \exp \left[-\frac{n^{2}}{1600}+j \frac{\pi n^{2}}{256} \tan (0.1 \pi)\right] \\
& y[n]=\frac{1}{2 \sqrt{\pi}} \exp \left[-\frac{n^{2}}{400}+j \frac{\pi n^{2}}{256} \tan (-0.3 \pi)\right]
\end{aligned}
$$

for $-128 \leq n<128$. Real parts of $x[n]$ and $y[n]$ are plotted in Fig. 2. The Wigner distribution of the corrupted signal is shown in Fig. 3, where we see that the interference $y[n]$ is much more dominant than the signal $x[n]$. We will employ the following strategy to recover the radar pulse. Recall that the axis making angle $a \pi / 2$ with the time/space axis is the ath fractional Fourier domain. Both the desired radar signal and the corrupting signal exhibit different degrees of compactness in different domains. Therefore we may transform to domains where their separation is relatively large and eliminate those parts which are heavily corrupted by the distorting signal and then use interpolation to recover the complete signal. We will make use of two domains, the domain in which the desired signal is maximally spread, and the domain in which it is most compact. The domain in which the desired signal is maximally spread is $a=0.2$, and the corrupted signal in this domain is shown in Fig. 4. We will eliminate the corrupted interval $-24 \leq n<24$ from this data and assume that the 0.2 th FRT of the signal is known only outside this interval, in addition to the support information $x[n]=0,|n| \geq 75$. Second, we look at the -0.8 th domain in which the desired radar pulse is most compact, shown in Fig. 5. Here, we know that the desired signal is of negligible value outside the interval $-4 \leq n<4$ so that we will assume the -0.8 th FRT of the signal is zero outside this interval. The signal is assumed not to be known inside the interval $-4 \leq n<4$. The available information in the two domains now fulfills Scenario 4 and the signal can be recovered by the interpolation procedure presented in this paper. The error falls to $0.2 \%$ after 200 iterations.

We also solved the same problem after adding additive zero-mean white Gaussian random noise to the corrupted radar pulse in the original $a=0$ th domain. The noise variance is $(0.002)^{2}$, which leads to

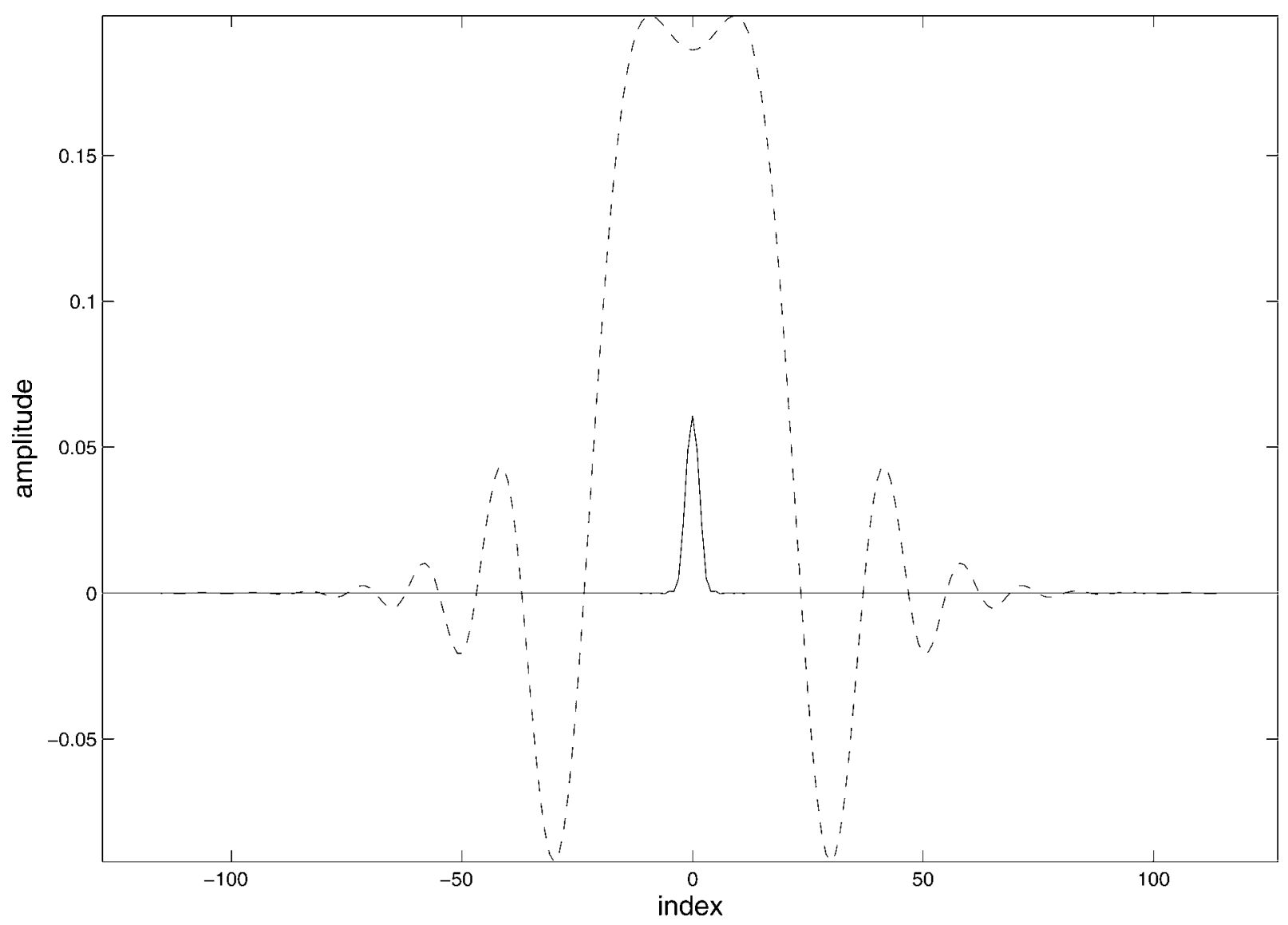

Figure 5 Real parts of the -0.8th fractional Fourier transforms of the radar pulse and interference signal Solid line: radar pulse; dashed line: interference signal 
noise sample values comparable to the uncorrupted signal. The error falls to around $2-3 \%$ after 50 iterations.

Although not used in this example, two additional domains could have been employed: (i) The domain in which the distorting signal is maximally distributed; here, we would have eliminated all samples outside of a centrally located interval. (ii) The domain in which the distorting signal is most compact; here, we would have eliminated the centrally located interval where the distorting signal is dominant.

The general nature of our formulation and the flexibility it affords allows it to be applied to situations where we need to recover a propagating wave from measurements from a random and/or irregular or otherwise inconveniently arranged array of sensors. Such problems arise with sensor networks, where a large number of possibly irregularly situated sensors may be employed to gather information on the environment [32, 33]. Similarly, the same problem may arise with swarm robots, where a large number of robots with relatively simple sensors and intelligence work cooperatively to understand and operate in a given environment [34, 35]. Based on the established relationship between the distance along the direction of propagation and the fractional order [1], a separate domain can be assigned to each measurement based on its axial coordinate. Only one sample value will be known and the others unknown in that domain. Therefore we are confronted with the problem of recovering a signal from a large number of domains, in each of which only one sample value is known. This problem is a special case of Scenario 4.

Let us consider the case where the 128 measurement points are randomly distributed both axially (in the propagation direction) and transversely. To keep the problem pure, we will assume the points are uniformly distributed both in $a$ and $u$. The distribution of points employed is shown in Fig. 6. With the support $[-5,25)$, the error drops below $0.12 \%$ after only 10 iterations and $0.0001 \%$ after 25 iterations. Let us now assume that the sample values are not known for every value of $a$ but for every other value, so that now there are a total of only 64 known samples instead of 128 . Despite this deficiency in the available information, the error drops below 2.2\% after 500 iterations.

Because of the theoretical assurance on algorithm convergence, there is no reason to think that the algorithm will behave differently with other examples.

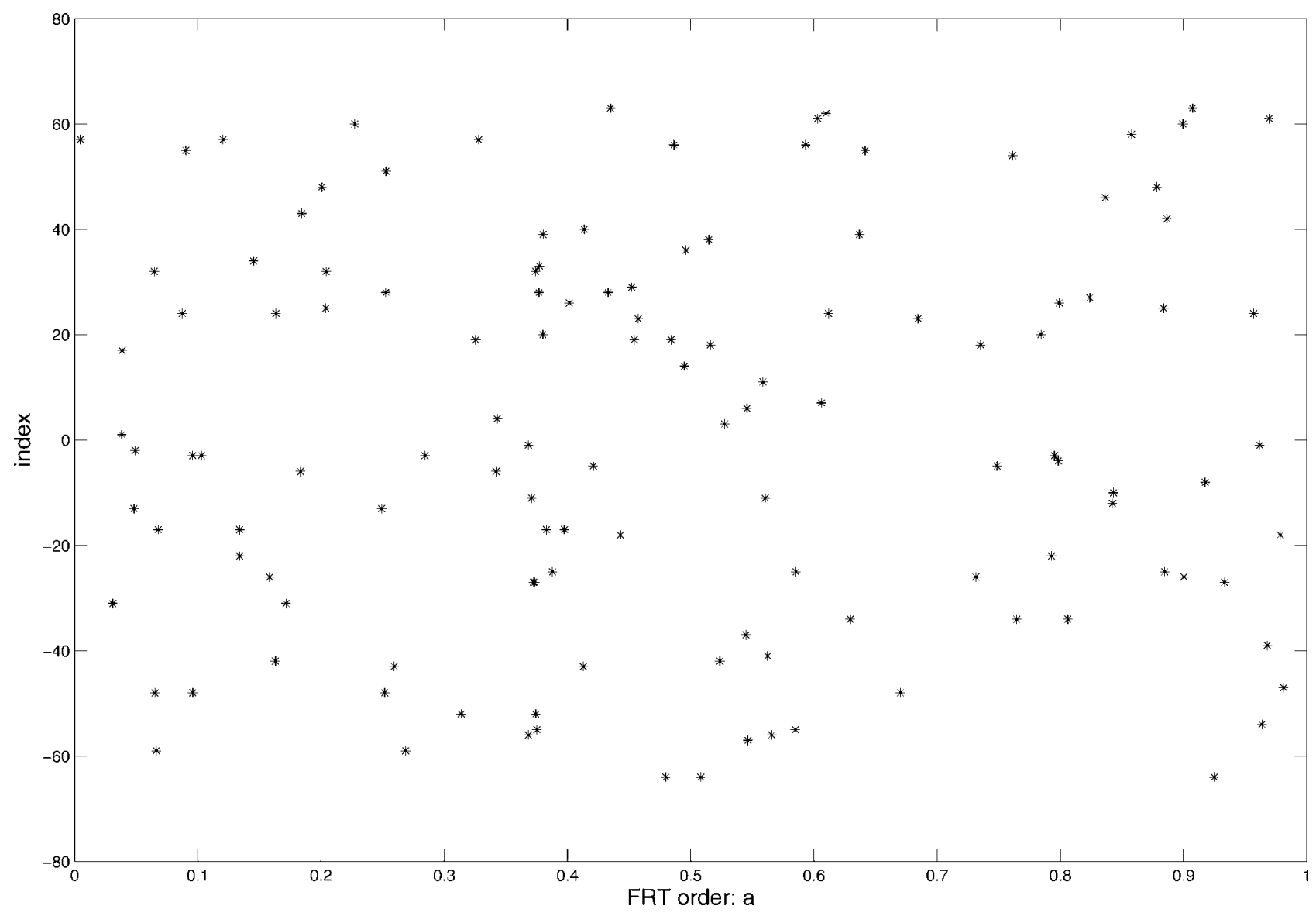

Figure 6 Distribution of known sample points 


\section{Discussion and conclusion}

In this paper, we discussed the solution of the problem of signal recovery from partial FRT domain information by using an iterative algorithm. This problem finds applications in wave propagation problems where the measured information is partial, spread over several observation planes, or not of sufficient spatial resolution. The signal recovery algorithm is based on the method of POCS and convergence is assured regardless of the initial estimate. After presenting the general formulation, we presented several generic application scenarios illustrating a wide variety of prototypical situations which are covered by our framework. We also presented an application example involving the recovery of a corrupted radar pulse and another example inspired by swarm robots and sensor networks. The presented framework can be easily extended to multi-dimensional problems as well. It can also be generalised to the case where signal information is available or can be deliberately measured in a number of generalised 'domains' which are related through linear transformations other than the FRT, such as the family of linear canonical transforms [36].

In all the examples considered, we have observed consistent behaviour of the algorithm. If the FRT measurements are available in a very narrow interval, the corresponding entries of the neighbouring rows of the transform matrix may get very close to each other and this structure may lead to unstable reconstruction results from noisy measurements. This is not especially related to the FRT case; in this respect, the problem is very similar to the problem of signal reconstruction from narrow-band ordinary Fourier transform information.

The relative overlap and separation of signal and noise (desired and undesired information), the localisation of this overlap, and therefore the signal-tonoise ratio at a certain interval will, in general, be different in different domains. By choosing regions in each domain where the signal-to-noise ratio is relatively favourable and discarding those regions where it is unfavourable and then using the generalised interpolation strategy presented in this paper to combine these partial signals, is a general approach which we believe will find widespread applicability in a variety of situations.

\section{Acknowledgment}

A preliminary version of this paper was presented at the $1 \mathrm{st}$ International Symposium on Control, Communications and Signal Processing, and was published by IEEE [19]. The authors would like to thank Laurence Barker of Bilkent University for useful suggestions. H.M. Ozaktas acknowledges the partial support of the Turkish Academy of Sciences.

\section{$7 \quad$ References}

[1] OZAKTAS HM, ZALEVSKY Z, KUTAY MA: 'The fractional Fourier transform with applications in optics and signal processing' (Wiley, New York, 2001)

[2] ozaktas hm, barshan B, Mendlovic D, et al.: 'Convolution, filtering and multiplexing in fractional Fourier domains and their relation to chirp and wavelet transforms', J. Opt. Soc. Am. A, 1994, 11, pp. 547-559

[3] ALMEIDA LB: 'The fractional Fourier transform and timefrequency representations', IEEE Trans. Signal Process., 1994, 42, pp. 3084-3091

[4] MENDLOVIC D, OZAKTAS HM: 'Fractional Fourier transforms and their optical implementation: I', J. Opt. Soc. Am. A, 1993, 10, pp. $1875-1881$

[5] OZAKTAS HM, MENDLOVIC D: 'Fractional Fourier transforms and their optical implementation: II', J. Opt. Soc. Am. A, 1993, 10, pp. 2522-2531

[6] ERDEN MF, KUTAY MA, OZAKTAS HM: 'Repeated filtering in consecutive fractional Fourier domains and its application to signal restoration', IEEE Trans. Signal Process., 1999, 47, pp. $1458-1462$

[7] KUTAY MA, ÖZAKTAŞ H, OZAKTAS HM, ET AL.: 'The fractional Fourier domain decomposition', Signal Process., 1999, 77, pp. 105-109

[8] Yetık iş, KUTAY MA, ÖZAKTAŞ H, ET Al.: 'Continuous and discrete fractional Fourier domain decomposition'. Proc. 2000 IEEE Int. Conf. Acoust, Speech, and Signal Processing, vol. I, 2000, pp. 93-96

[9] KUTAY MA, OZAKTAS HM: 'Optimal image restoration with the fractional Fourier transform', J. Opt. Soc. Am. A, 1998, 15, pp. 825-833

[10] BARSHAN B, AYRULU B: 'Fractional Fourier transform preprocessing for neural networks and its application to object recognition', Neural Netw., 2002, 15, pp. $131-140$

[11] OZAKTAS HM, ARIKAN O, KUTAY MA, ET AL.: 'Digital computation of the fractional Fourier transform', IEEE Trans. Signal Process., 1996, 44, pp. 2141-2150

[12] Yetik iş, OzAKTAS hM, BARShan B, ET Al.: 'Perspective projections in the space-frequency plane and fractional Fourier transforms', J. Opt. Soc. Am. A, 2000, 17, pp. $2382-2390$

[13] OZAKTAS HM, AYTÜR O: 'Fractional Fourier domains', Signal Process., 1995, 46, pp. 119-124 
[14] BARKER L, CANDAN ç, HAKIOĞLU T, ET AL.: 'The discrete harmonic oscillator, Harper's equation, and the discrete fractional Fourier transform', J. Phys. A, 2000, 33, pp. 2209-2222

[15] TAO R, DENG B, WANG Y: 'Research progress of the fractional Fourier transform in signal processing', Sci. China (Ser. F, Inf. Sci.), 2006, 49, pp. 1-25

[16] OZAKTAS HM, MENDLOVIC D: 'Fractional Fourier optics', J. Opt. Soc. Am. A, 1995, 12, pp. 743-751

[17] OZAKTAS HM, MENDLOVIC D: 'Fractional Fourier transform as a tool for analyzing beam propagation and spherical mirror resonators', Opt. Lett., 1994, 19, pp. 1678-1680

[18] ERDEN MF, OZAKTAS HM, MENDLOVIC D: 'Synthesis of mutual intensity distributions using the fractional Fourier transform', Opt. Commun., 1996, 125, pp. 288-301

[19] CETIN AE, ÖZAKTAŞ H, OZAKTAS HM: 'Signal recovery from partial fractional Fourier transform information'. Proc. 1st Int. Symp. Control, Communications and Signal Processing (ISCCSP 2004), March 2004, pp. 217-220

[20] YOULA DC, WEBB H: 'Image restoration by the method of convex projections, part-1: Theory', IEEE Trans. Med. Imaging, 1982, MI-1, (2), pp. 81-94

[21] STARK H: 'Image recovery - theory and application' (Academic Press, Orlando, 1987)

[22] COMBETTES PL: 'The foundations of set theoretic estimation', Proc. IEEE, 1993, 81, pp. 182-208

[23] COMBetTes PL: 'The convex feasibility problem in image recovery'. Advances in Imaging and Electron Physics, vol. 95, (Academic Press, 1996), pp. 155-270

[24] CANDAN Ç, KUTAY MA, OZAKTAS HM: 'The discrete fractional Fourier transform', IEEE Trans. Signal Process., 2000, 48, pp. 1329-1337
[25] CANDAN Ç: 'Discrete fractional Fourier transform matrix generator' MATLAB program, 1998. Available at http:// www.ee.bilkent.edu.tr/_haldun/dFRT.m

[26] SEZAN MI, STARK H: 'Image restoration by the method of convex projections, part-2: Applications and numerical results', IEEE Trans. Med. Imaging, 1982, MI-1, (2), pp. 95-101

[27] TRUSSELL HJ, CIVANLAR MR: 'The feasible solution in signal restoration', IEEE Trans. Acoust. Speech Signal Process, 1984, 32, pp. 201-212

[28] CETIN AE, ANSARI R: 'A convolution based framework for signal recovery', J. Opt. Soc. Am. A, 1988, 5, pp. 1193-1200

[29] CETIN AE, ANSARI R: 'Signal recovery from wavelet transform maxima', IEEE Trans. Signal Process., 1994, 42, pp. 194-196

[30] CETIN AE, GEREK ON, YARDIMCI Y: 'Equiripple FIR filter design by the FFT algorithm', IEEE Signal Process. Mag., 1997, 14, pp. 60-64

[31] CETIN AE, ÖZAKTAŞ H, OZAKTAS HM: 'Resolution enhancement of low resolution wavefields with POCS algorithm', Electron. Lett., 2003, 39, pp. 1808-1810

[32] AKYildiz IF, Weilian S, sankarasubramaniam Y, et al.: 'A survey on sensor networks', IEEE Commun. Mag., 2002, 40, pp. $102-114$

[33] IYENGAR SS, BROOKS RR: 'Distributed sensor networks' (Chapman \& Hall/CRC Press, 2004)

[34] DORIGO M, SAHIN E (EDS): 'Guest editorial on Swarm robotics', Auton. Robots, 2004, 17, pp. 111-113

[35] SAHIN E, SPEARS W (EDS): 'Swarm robotics: state-of the-art survey', Lect. Notes Comput. Sci., 2005, 3342

[36] BARSHAN B, KUTAY MA, OZAKTAS HM: 'Optimal filtering with linear canonical transformations', Opt. Commun, 1997, 135, pp. 32-36 